\title{
Influencing the Angular Emission of a Single Molecule
}

\author{
H. Gersen,* M. F. García-Parajó, L. Novotny, ${ }^{\dagger}$ J. A. Veerman, L. Kuipers, and N. F. van Hulst \\ Applied Optics Group, Department of Applied Physics \& MESA $A^{+}$Research Institute, University of Twente, P.O. Box 217, \\ 7500 AE Enschede, The Netherlands
}

(Received 5 July 2000)

\begin{abstract}
We present the first experimental proof for the influence of a nearby nanosized metal object on the angular photon emission by a single molecule. Using a novel angular sensitive detection scheme, we directly quantify the redirection of angular emission for different molecular dipole orientations as an object is scanned laterally over the molecule at different heights. An excellent agreement between experiments and 2D-numerical simulations is found for molecules oriented perpendicular to the sample, whereas, for parallel orientations, the observed behavior contradicts the calculated behavior.
\end{abstract}

PACS numbers: 33.50.-j, 07.79.Fc, 42.25.Gy, 78.66.-w

In many molecular fluorescence experiments the molecules of interest are located in close proximity to an interface between dielectric or metallic materials. In a series of beautiful experiments by Drexhage [1] in the early 1970s, it was shown that the fluorescence lifetime of excited molecules sensitively depends on the distance to a nearby metal surface. These observations demonstrate that the molecular emission properties depend on the local environment. Although full quantum electrodynamic calculations [2] have been carried out to interpret the influence of the local boundary conditions on the molecular emission properties, much of the physics involved can be addressed by a phenomenological theory $[3,4]$. In this approach, the quantum mechanical transition dipole is replaced by a classical dipole driven by the local electromagnetic field. The experiments by Drexhage and others were, however, limited to the investigation of ensembles of emitters at planar interfaces. As a consequence of the resulting average over different dipole orientations and large sample areas any possible effect of the breaking of the planar geometry on the molecular emission remained hidden. To study the influence of nanometer-sized objects, which break the planar geometry, on the molecular emission properties one has to overcome the limitations of the early experiments by investigating single emitters. The influence of nanometer-sized objects has recently gained interest due to advances in manipulation of spontaneous emission in nanometric structures [5,6], the observation of enhanced fluorescence at sharp metal tips [7], and Raman scattering at nanometric particles due to high local fields [8].

In 1993, Betzig and Chichester [9] were the first to image fluorescence emission of single emitters under ambient conditions using an aperture-type near-field scanning optical microscope (NSOM). The individual molecules were immobilized on a planar substrate and locally excited with a metal-coated light emitting probe that was scanned in close proximity to the sample surface. By controlling the polarization of the excitation they managed to determine the dipole orientation for each molecule in three dimensions. Obviously, NSOM thus enables the study of the interaction of a single emitter with its local environment in a controllable way for all molecular orientations [10]. Indeed, in measuring fluorescence lifetimes of individual molecules using NSOM, different groups observed the dependence of molecular lifetimes on both lateral displacement of the metallized probe and probe-sample separation [11-13]. The experimental observations were verified with numerical simulations in which a finite-sized object (the NSOM probe) is scanned laterally over the molecule at different heights. In doing so, it was shown $[14,15]$ that the fluorescence lifetime of a single emitter is highly dependent both on the orientation of the dipole and on the relative position of a finite-sized object. Effects on the lifetime are small $(<10 \%)$ when the probe is centered above the molecule, but near the edges of the aperture the lifetime changes dramatically by at least a factor of $3[11-15]$. Near the edges of a finite-sized metal object it was predicted [15] that not only lifetime but also the angular emission of a single emitter should be strongly affected. The presence of the object would cause a redirection of the angular emission towards the metal object.

In this Letter, we present the first experimental proof for the redirection of the angular emission of an individual molecule as induced by the close proximity of a nanometer-sized metal object. Using a novel detection scheme, we directly quantify the degree of angular emission pattern modification for molecules with dipole moments oriented, respectively, perpendicular and parallel (in-plane) to the sample surface as the object is scanned laterally over the molecule at different heights. The observed effects are compared with calculations based on the multiple multipole (MMP) technique.

To investigate the effect of a nanosized metal object on the single molecule emission pattern it is essential to detect the emission as a function of the emission angle. For this purpose we implemented a novel experimental scheme exploiting the fact that the fluorescence emitted by the molecule at a given angle in the focus of an objective emerges at a well-defined position at the back focal plane of that objective. A specific area of this plane is directed to a detector, giving information concerning the emission 
at angles corresponding to that area. To obtain maximum sensitivity for asymmetric emission and still have sufficient photons to allow single molecule detection we separate the emission at the optical axis in two different parts given by the $-x$ and $+x$ half space (see Fig. 1).

The separation in two different half spaces is accomplished by inserting a reflecting knife-edge parallel to the $y$ axis and directing each half space onto a separate photon-counting avalanche photodiode. The insertion of a knife-edge along the $y$ axis results in a method exclusively sensitive for asymmetries in the $x-z$ plane ( $z$ is the optical axis). The reflection layer on the knife-edge is made by evaporating a thin layer of silver on a microscope cover slide $(76 \times 23 \mathrm{~mm}$, cut edges) resulting in a reflection coefficient of $98.0 \% \pm 0.5 \%$ and $94 \% \pm 1 \%$ for $s$ and $p$ polarization, respectively. We use the excitation light emitted by the NSOM probe to align the experimental setup. Subsequently, the angular emission can be split in two equal parts with an accuracy of better than $1.5 \%$ as determined from the background signal on the two detectors, originating mainly from the luminescence of the fiber.

For the experiment itself, we bring a nanometer-sized metal object in close proximity to individual molecules. The aluminum coating of the optical probe of an aperturetype NSOM appears predestined for this purpose. The positioning accuracy of an NSOM $(\sim 1 \mathrm{~nm})$ allows a systematic investigation of the intensity ratio between two different half spaces as a function of the position of the metal-glass interfaces with respect to the single emitter. The new detector configuration (Fig. 1) has been implemented in an existing NSOM setup that is described in detail elsewhere [16]. The near-field optical probes in our experiment are modified by side-on milling with a focused ion beam (FIB), as introduced by us [17]. We obtain probes with a flat end face (surface roughness $<1.5 \mathrm{~nm}$ ) and a well-defined circular aperture with a radius of $\sim 80 \mathrm{~nm}$

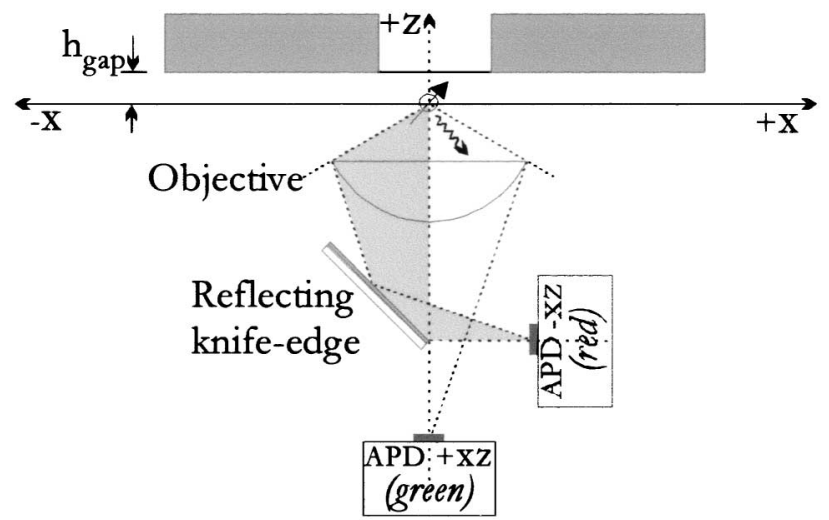

FIG. 1. Detection scheme for angle specific detection. Fluorescence emitted at a given solid angle corresponds to a specific area in the back focal plane of the objective. The knife-edge in that plane, placed parallel to the $y$ axis (out of paper), separates the emission in the $-x$ and $+x$ half spaces.
[18]. The edge sharpness of the metal-glass interface is solely determined by the optical penetration depth of the metal $(6 \mathrm{~nm}$ for $\mathrm{Al})$.

Single-molecule samples are prepared by spin-coating carbocyanine dye $\left(\mathrm{DiIC}_{18}\right)$ molecules in solution with polymethyl-methacrylate (PMMA, $10^{-9} \mathrm{M}$ ) onto a glass cover slip. The well-separated single molecules are excited with linearly (both $x$ - and $y$-) and circularly polarized light $(\lambda=514 \mathrm{~nm})$ as determined in the far field. Figure 2 shows a set of angle specific single molecule images in which a false-color scale is applied that represents the relative contribution of the two half spaces to the collected power. Red and green are used for the power emitted to the half spaces given by the negative and positive $x$ directions, respectively; an equal signal on both detectors results in a yellow color.

Figure 2(a) shows a typical measurement for circularly polarized excitation light. Several important observations can be made. First, the observed near-field excitation patterns correspond well to the expected intensity patterns of the Bethe-Bouwkamp model $[9,16,19,20]$ generated by the overlap of the molecular absorption dipole moment with the electrical fields close to the aperture. This overlap at a certain position of the aperture determines the excitation rate of the molecule. The in-plane molecules are preferentially excited when they are centered beneath the near-field probe while the $z$-oriented molecules are excited mainly

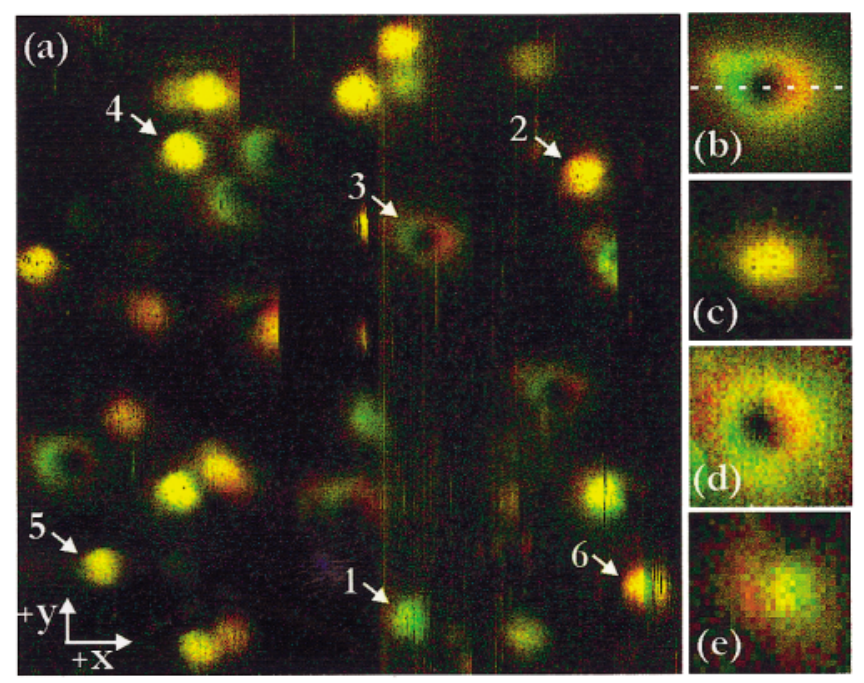

FIG. 2 (color). Angle specific NSOM fluorescence images obtained with an $\sim 80 \mathrm{~nm}$ aperture probe showing DiIC $_{18}$ molecules embedded in a $10 \mathrm{~nm}$ thin film of PMMA (all images circularly polarized excitation, scan direction along $y$ ). The false-color scale (red/green) indicates emission contribution into the $-x$ and $+x$ half spaces, respectively. An equal signal on both detectors results in a yellow color. (a) Scan size $2.4 \times$ $2.4 \mu \mathrm{m}^{2}, h_{\text {gap }}=20 \pm 5 \mathrm{~nm}$. (b) $-(\mathrm{e})$ Detailed scans $(400 \times$ $400 \mathrm{~nm}^{2}$ ) of individual molecules: (b) $z$-oriented molecule, $h_{\text {gap }}=20 \pm 5 \mathrm{~nm} ; \quad$ (c) $x$-oriented molecule, $h_{\text {gap }}=$ $20 \pm 5 \mathrm{~nm}$; (d) $z$-oriented molecule, $h_{\text {gap }}=60 \pm 10 \mathrm{~nm}$; (e) $x$-oriented molecule, $h_{\text {gap }}=60 \pm 10 \mathrm{~nm}$. 
close to the aperture rims. In the latter case, for example, the molecular excitation patterns show up as circles when circularly polarized excitation light is used. From the lateral dimensions of the excitation patterns the probe-sample separation is estimated to be $20 \pm 5 \mathrm{~nm}$. Second, overall "color" differences between different molecules can be distinguished as can be clearly seen by comparing molecules 1 and 2. The asymmetric distribution of the total amount of photons emitted by a molecule between the $-x$ and $+x$ half space indicates an out-of-plane component of the emission dipole. For molecules with given fixed emission dipole orientation a fixed asymmetry (and thus color) is therefore expected.

However, one can clearly see color variations within the near-field excitation pattern, e.g., molecule 3, that indicate a redistribution of the emitted photons over the two half spaces as the probe is scanned over the molecule. Any influence of the probe on the molecular emission is expected to be strongly dependent on the position of the probe with respect to the molecule. Therefore, we attribute the observed redistribution of radiation to the presence of the metal-glass transitions of the probe. The observed effects are particularly strong for molecules with a significant $z$ component, e.g., molecule 3 , whereas for molecules 4 and 5 with mainly an in-plane orientation the effect is less pronounced.

Figures 2(b) and 2(c) display detailed scans on specific molecules $\left(400 \times 400 \mathrm{~nm}^{2}\right)$ for a $z$ - and an $x$-oriented molecule at a probe-sample separation of $20 \pm 5 \mathrm{~nm}$, respectively. The molecular orientations are determined directly from the excitation patterns and the fact that the total amount of photons emitted between the $-x$ and $+x$ half spaces has to be symmetric. Figures 2(d) and 2(e) display the same set of orientations for a probe-sample separation of $60 \pm 10 \mathrm{~nm}$. It is clear that the emission redistribution observed for $z$-oriented molecules is not very sensitive to the probe-sample separation. However, for $x$-oriented molecules a dramatic height dependence is evident: at a height of $20 \mathrm{~nm}$ no effect of the metal is observable, but at $60 \mathrm{~nm}$ a clear redistribution of the emission is seen. In Fig. 3 we present a cross section through the excitation pattern of the $z$-oriented molecule taken at the dashed line in Fig. 2(b). The double lobe structure results from the strong $z$ component of the excitation field at the aperture rims. Figure 3 shows that when the molecule is right beneath the aperture rim, one detector detects 3-4 times more photons than the other detector. The observed asymmetry as induced by the probe is much larger than the asymmetry resulting from an out-of-plane component of the molecular transition dipole [cf. molecules 1 and 2 in Fig. 2(a), which show maximum observed asymmetry]. As the observed asymmetric emission is reproducible, we can also exclude the possibility of an emission-dipole reorientation as a function of the lateral position of the probe. It is possible to observe some rotational dynamics, e.g., molecule 6 in Fig. 2(a). For this molecule we observe discrete jumps

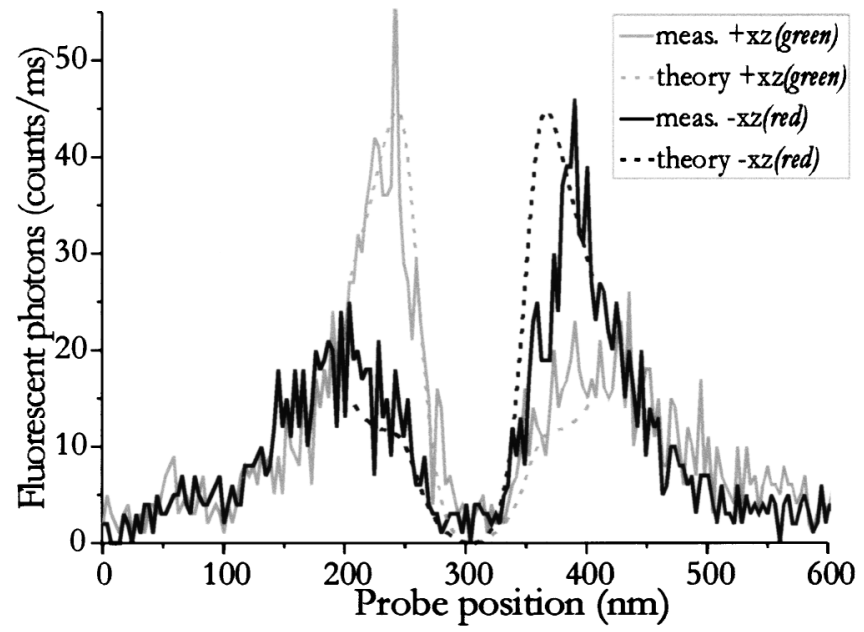

FIG. 3. The fluorescence of both half spaces displayed separately (solid curves) for the $z$-oriented molecule in Fig. 2(b) (at dashed line) for linear $x$-polarized excitation. Maximum excitation of this molecule occurs at the gradient field around the metal-glass transitions. Clearly 3-4 times more signal is collected in one half-space with respect to the other half-space below the aperture rims. Dotted curves present the result of numerical simulation $\left(h_{\mathrm{gap}}=20 \mathrm{~nm}\right)$ showing the excellent agreement between theory and experiment.

between different $z$ components. Molecules with such a dynamic behavior have been excluded from our analysis.

In our experiment, the scan movement of the sample stage is such that the images can be viewed as if the probe is moving across the image while scanning. For both $z$ - and $x$-oriented molecules, Fig. 4 schematically depicts the relative probe position and the corresponding direction of the probe-induced effect on the emission pattern. The sequence (a)-(e) corresponds to a movement of the probe from $-x$ to $+x$. Although the $z$-oriented molecule in Fig. 4(a) is not strongly excited, it can be determined that the fluorescence emission is directed towards the $-x$ half space [more "red," Fig. 4(a)]. As the near-field probe scans further, the molecule is strongly excited at the metal-glass interface on the $+x$ side of the probe [Fig. 4(b)]. Now a redirection of the fluorescence towards the $+x$ half space [more "green," Fig. 4(b)] is observed. When the molecule is centered beneath the probe [Fig. 4(c)] the emission is symmetric. As the scan process continues, the redirection of the angular emission is repeated in reverse [Figs. 4(d), 4(e)]. Thus, it can be concluded that the fluorescence photons of a $z$-oriented molecule are attracted towards the center of the aluminum coating. The above mentioned effect describes the measurements presented in Figs. 2(b), 2(d), and 3. A similar comparison for $x$-oriented molecules [Figs. 4(a)-4(e)] shows that the fluorescence photons are repelled from the aluminum coating towards the center of the aperture. Hence, we find that the angular redistribution shows a distinct dependence on the orientation of the emission dipole of the molecule. 


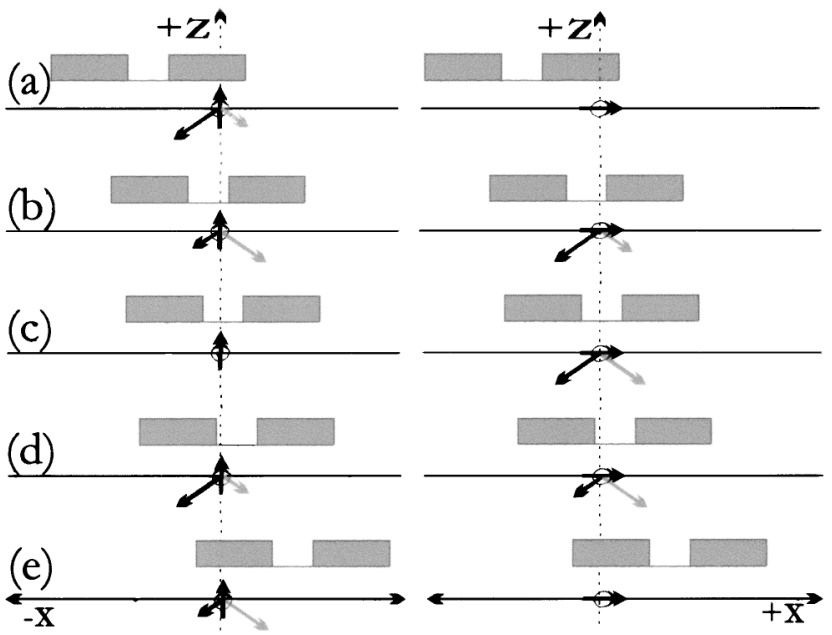

FIG. 4. Schematic illustration of the relative position of the probe during the scan for both a $z$-oriented dipole (left column) and an $x$-oriented dipole (right column). Sequence (a)-(e) corresponds to a tip movement from $-x$ to $+x$. Length of the depicted arrows indicates the relative amount of power emitted into each half space. A larger relative contribution in the $-x$ and the $+x$ half space correspond to "red" and "green" in Fig. 2, respectively. For $z$-oriented dipoles the metal attracts the emitted photons, whereas for $x$ orientation the emitted photons are repelled from the metal.

The observed distance dependence of $x$-oriented molecules can be explained by the fact that these molecules are excited mainly at the center of the aperture. For a $20 \mathrm{~nm}$ separation the effect of the metal-glass interface is not clearly observable as the molecule is not sufficiently excited below the rim. For the larger separation of $60 \mathrm{~nm}$ the $x$-oriented molecule is excited also partly below the rim as can be seen clearly from the larger extension of the excitation pattern in Fig. 2(e) with respect to Fig. 2(c).

To confront the observations with theory, calculations based on the MMP technique have been carried out for a 2D geometry as depicted in Fig. 1. In these calculations, the molecule is modeled as a classical dipole. The radiation field of the dipole is evaluated in both half spaces as a function of the relative dipole-probe position. In order to calculate the position dependent angular emission the excitation field of the near-field probe is included without considering quenching. The result given in Fig. 3 shows excellent agreement between theory and experiment for z-oriented dipoles without any fit to the experimental data. The conflicting results for theory and experiment for $x$-oriented dipoles, however, indicate that a full three-dimensional calculation is unavoidable to explain the observations completely.
In summary, for the first time we have observed the influence of a nanometer-sized object on the angular emission of single molecules. Because of the close proximity of sharp well-defined material boundaries of FIB modified NSOM probes, the angular emission of a single molecule is strongly modified. For $z$-oriented molecules the emitted photons are attracted toward the center of the metal coating in agreement with 2D-MMP calculations. For $x$-oriented molecules an influence is observed only for larger probe-sample separations. In this case we find that the emitted photons are repelled from the metal in contrast to the calculated behavior. The strength of the observed effect has severe implications for current efforts to obtain near-field optical imaging based on local field enhancement by sharp metal tips and the determination of the 3D orientation of emitters in a heterogeneous environment.

*Electronic address: h.gersen@tn.utwente.nl

†Present address: The Institute of Optics, University of Rochester, Wilmot 519, Rochester, NY 14627.

[1] For a review, see K.H. Drexhage, Prog. Opt. 12, 163 (1974).

[2] P.W. Milonni, The Quantum Vacuum (Academic, San Diego, 1994).

[3] For a review, see R. R. Chance, A. Prock, and R. Silbey, Adv. Chem. Phys. 37, 1 (1978).

[4] W. Lukosz, Phys. Rev. B 22, 3030 (1980).

[5] E. Yablonovitch, Phys. Rev. Lett. 58, 2059 (1987).

[6] M. Megens, J. E. G. J. Wijnhoven, A. Lagendijk, and W. L. Vos, Phys. Rev. A 59, 4727 (1999).

[7] E. J. Sánchez, L. Novotny, and X. S. Xie, Phys. Rev. Lett. 82, 4014 (1999).

[8] S. Nie and S. R. Emory, Science 275, 1102 (1997).

[9] E. Betzig and R. J. Chichester, Science 262, 1422 (1993).

[10] J. A. Veerman, M. F. Garcia-Parajo, L. Kuipers, and N. F. van Hulst, Phys. Rev. Lett. 83, 2155 (1999).

[11] W. P. Ambrose, P. M. Goodwinn, J.C. Martin, and R. A. Keller, Science 265, 364 (1994).

[12] X. S. Xie and R. C. Dunn, Science 265, 361 (1994).

[13] J. K. Trautman and J. J. Macklin, Chem. Phys. 205, 221 (1996).

[14] R. X. Bian, R. C. Dunn, and X. S. Xie, Phys. Rev. Lett. 75, 4772 (1995).

[15] L. Novotny, Appl. Phys. Lett. 69, 3806 (1996).

[16] A. G. T. Ruiter, J.A. Veerman, M.F. Garcia-Parajo, and N. F. van Hulst, J. Phys. Chem. A 101, 7318 (1997).

[17] J. A. Veerman, A. M. Otter, L. Kuipers, and N. F. van Hulst, Appl. Phys. Lett. 72, 3115 (1998).

[18] J. A. Veerman, M.F. Garcia-Parajo, L. Kuipers, and N. F. van Hulst, J. Microsc. 194, 477 (1999).

[19] H. A. Bethe, Phys. Rev. 66, 163 (1944).

[20] C. H. Bouwkamp, Philips Res. Rep. 5, 321 (1950). 